\title{
Production and perception of French vowels by congenitally blind adults and sighted adults
}

Lucie Ménard, Sophie Dupont, Shari R. Baum, and Jérôme Aubin

Citation: The Journal of the Acoustical Society of America 126, 1406 (2009); doi: 10.1121/1.3158930

View online: https://doi.org/10.1121/1.3158930

View Table of Contents: https://asa.scitation.org/toc/jas/126/3

Published by the Acoustical Society of America

\section{ARTICLES YOU MAY BE INTERESTED IN}

Acoustic and articulatory analysis of French vowels produced by congenitally blind adults and sighted adults The Journal of the Acoustical Society of America 134, 2975 (2013); https://doi.org/10.1121/1.4818740

Vowel production in sighted children and congenitally blind children

The Journal of the Acoustical Society of America 136, 2105 (2014); https://doi.org/10.1121/1.4899575

Congenital blindness enhances perception of musical rhythm more than melody in Mandarin speakers The Journal of the Acoustical Society of America 145, EL354 (2019); https://doi.org/10.1121/1.5100899

Visual Contribution to Speech Intelligibility in Noise

The Journal of the Acoustical Society of America 26, 212 (1954); https://doi.org/10.1121/1.1907309

Production of French vowels by American-English learners of French: Language experience, consonantal context, and the perception-production relationship

The Journal of the Acoustical Society of America 128, 1290 (2010); https://doi.org/10.1121/1.3466879

Articulatory and acoustic adaptation to palatal perturbation

The Journal of the Acoustical Society of America 129, 2112 (2011); https://doi.org/10.1121/1.3557030

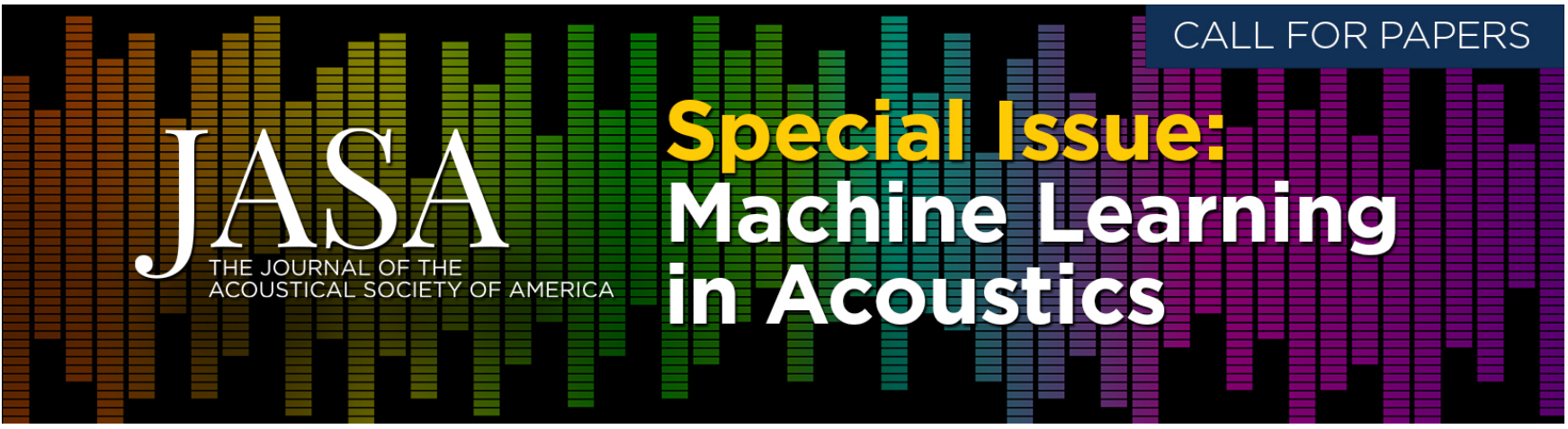




\title{
Production and perception of French vowels by congenitally blind adults and sighted adults
}

\author{
Lucie Ménard $^{\text {a) }}$ and Sophie Dupont \\ Département de Linguistique et de Didactique des Langues, Laboratoire de Phonétique, \\ Center for Research on Language, Mind, and Brain, Université du Québec à Montréal, Montreal, \\ Quebec H3C 3P8, Canada \\ Shari R. Baum \\ School of Communication Sciences and Disorders, Center for Research on Language, Mind, and Brain, \\ McGill University, Montreal, Quebec H3G 1A8, Canada \\ Jérôme Aubin \\ Département de Linguistique et de Didactique des Langues, Laboratoire de Phonétique, \\ Center for Research on Language, Mind, and Brain, Université du Québec à Montréal, Montreal, \\ Quebec H3C 3P8, Canada
}

(Received 6 August 2008; revised 18 May 2009; accepted 30 May 2009)

\begin{abstract}
The goal of this study is to investigate the production and perception of French vowels by blind and sighted speakers. 12 blind adults and 12 sighted adults served as subjects. The auditory-perceptual abilities of each subject were evaluated by discrimination tests (AXB). At the production level, ten repetitions of the ten French oral vowels were recorded. Formant values and fundamental frequency values were extracted from the acoustic signal. Measures of contrasts between vowel categories were computed and compared for each feature (height, place of articulation, roundedness) and group (blind, sighted). The results reveal a significant effect of group (blind vs sighted) on production, with sighted speakers producing vowels that are spaced further apart in the vowel space than those of blind speakers. A group effect emerged for a subset of the perceptual contrasts examined, with blind speakers having higher peak discrimination scores than sighted speakers. Results suggest an important role of visual input in determining speech goals. () 2009 Acoustical Society of America. [DOI: $10.1121 / 1.3158930]$
\end{abstract}

PACS number(s): 43.70.Mn, 43.71.Es [RSN]

Pages: $1406-1414$

\section{INTRODUCTION}

In the past decades, several studies have shown that visual cues provided by the lips and jaw are not simply redundant in the process of speech perception; in fact, they act as functional cues that supplement the auditory information transmitted by the speech signal (McGurk and MacDonald, 1976; Robert-Ribes et al., 1998). In the audiovisual modality, speech intelligibility scores are higher than in the auditory modality alone or in the visual modality alone (Grant et al., 1998; Reisberg et al., 1987). The role played by visual cues in speech perception is crucial for perceivers without access to auditory input (Andersson et al., 2001; Bernstein et al., 2001). However, perceptual cues conveyed by the visual channel alone do not allow the listener to recover all the phonological contrasts of a language, as revealed by the fact that prelingually deaf speakers without hearing aids never fully gain the ability to perceive speech on the basis of speechreading alone (cf. Bernstein et al., 2000).

Although the visual modality is crucial for deaf speakers, the fact that congenitally blind speakers learn to produce correct speech sounds suggests that visual cues are not mandatory in the control of speech movements. Nevertheless,

\footnotetext{
a) Author to whom correspondence should be addressed. Electronic mail: menard.lucie@uqam.ca
}

several studies conducted with blind speakers revealed that their speech discrimination abilities differ from those of sighted speakers (Lucas, 1984; Hugdahl et al., 2004; Gougoux et al., 2004). This difference in auditory discrimination abilities between the groups may reflect differences at the level of production, given that the ability to discriminate speech sounds has been suggested to be related to individual differences in the amount of articulatory-acoustic contrast produced between the two sounds (Perkell et al., 2004, for instance). The links between production and perception have been evidenced in hearing and sighted subjects. For example, in 11 subjects, speaker's judgments of synthetic vowel similarities were correlated with that speaker's produced formant values for corner vowels. Newman (2003) also found production-perception relationships in subjects' produced and perceived voicing onset times values. Bell-Berti et al. (1979) and Perkell et al. (2004) reported electromyographic (EMG) (for the former) and articulatory data (for the latter) significantly related to the subjects' perceptual abilities. Other studies have shown parallel changes in production and perception dimensions, such as Bradlow et al. (1997), Rvachew (1994), and Vick et al. (2001) for cochlear implant subjects.

Furthermore, apart from differences in discrimination abilities between congenitally blind speakers and sighted speakers, the lack of access to visual information might also 
induce differences in the use and/or control of the speech articulators (especially the visible ones). To the best of our knowledge, no study has addressed speech production abilities in adult speakers with visual impairments.

This paper aims to investigate auditory discrimination abilities and the production of vowel contrasts in 12 congenitally blind adults and 12 sighted adults, all native speakers of Canadian French. Vowels were chosen because they are perceptually salient in the speech stream and tend to yield relatively consistent percepts.

\section{AUDITORY PERCEPTION IN BLIND SPEAKERS}

Without visual information, blind speakers rely solely on the auditory signal to recover phonological information. In a review of studies conducted on blind and sighted speakers between 1960 and 1980, Miller (1992) showed that studies have produced somewhat contradictory results regarding auditory acuity in the two speaker groups. Stankov and Spilsbury (1978), for instance, studied rhythm perception and frequency discrimination in music and speech in clear and distorted (background noise or reduced tempo) conditions in 30 young speakers (between 10 and 15 years of age) belonging to three groups: totally blind, partially blind, and sighted. Blind speakers performed better than sighted speakers in frequency discrimination tasks, but no difference was found between the two groups in speech identification tasks in distorted conditions. Starlinger and Niemeyer (1981) and Niemeyer and Starlinger (1981) also conducted a series of perceptual experiments on blind and sighted adults. They found no difference in frequency discrimination thresholds, intensity discrimination thresholds, or duration discrimination thresholds. Despite the fact that those low-level tasks were performed equally well by both groups, in higher-level identification tasks, such as binaural integration of pure tones and noise, the blind speakers performed significantly better than the sighted speakers. Lucas (1984), Hugdahl et al. (2004), and Gougoux et al. (2004) also found superior non-speech auditory perceptual abilities in blind speakers. It should be mentioned, however, that the blind speaker groups in these studies were heterogeneous in many respects, for instance, concerning speaker age, age at blindness, degree of blindness, etc. Such variability is confounded with visual impairment and could have greatly influenced the results.

\section{SPEECH PRODUCTION IN BLIND SPEAKERS}

As reported by Kuhl and Meltzoff (1982) and Legerstee (1990), by the age of 4 months, sighted babies demonstrate strong capacities to associate sounds with the corresponding visual representation of the lips. Babies also imitate labial movements of sounds visually presented. It seems that at this language acquisition stage, babies recognize relationships between auditory parameters and visual events. Although most of the studies addressing auditory perceptual abilities in blind speakers have been conducted with adult subjects, speech production has been mainly described for blind children. As Elstner (1983) stated, visual impairment deprives the child of an important source of information that may have consequences for the strategies used to produce phono- logical targets. At the pre-babbling stage, Lewis (1975) reported less imitation of labial speech gestures by a blind baby compared to sighted babies. Elstner (1983) and Mills (1987) presented various studies showing phonological delays and phonetic/phonological disorders in older children. In a study of syllables produced by a small number of congenitally blind children (1-2 years of age), Mills (1987) reported a higher number of phonological confusions between groups of visually dissimilar consonants (labial/b/ vs velar $/ \mathrm{k} /$ ) for the blind children compared to sighted children. These data must, however, be interpreted with caution since they come from a very small sample. Furthermore, as reported by Elstner (1983), it is difficult to study homogeneous populations of blind speakers, and observed differences in speech production abilities between blind and sighted groups might just as well be related to the presence of uncontrolled variables, such as additional motor control disorders or language disorders, unrelated to the visual impairment.

In perhaps the most directly relevant study, Göllesz (1972) collected EMG data from 13-year-old and 14-year-old blind Hungarian male speakers uttering vowels. Sighted control subjects were also recorded. Despite reduced labial dynamics in blind speakers compared to sighted speakers, as measured by the degree of EMG activation, no significant differences were observed in the acoustic signal. These results suggest that visual impairment leads speakers to adopt different control strategies for the visible labial articulators. Some compensatory abilities of the other articulators are also likely involved to offset the limited movements of the lips to reach the acoustic target.

The objectives of the present study are the following. First, auditory discrimination abilities along the three phonological contrasts in French oral vowels (height, place of articulation, and roundedness) are investigated in 12 congenitally blind adults and 12 sighted adults. Second, the production of the French oral vowels by both groups of speakers in the acoustic space in terms of between-category contrast distances is studied. Third, production-perception relationships are analyzed through multiple regression analyses.

\section{METHOD}

\section{A. Participants}

12 congenitally blind adults ( 6 males and 6 females) and 12 sighted adult control subjects (6 males and 6 females) participated in the study. All subjects were native speakers of Canadian French living in the Montreal area. (Although the majority had some exposure to English, all use French as their primary language.) The blind speakers had a congenital and complete visual impairment, classified as class 3,4 , or 5 in the International Disease Classification of the World Health Organization. They had never had any perception of light or movement. They ranged in age from 26 to 52 years (mean: 44). They did not demonstrate any language disorders or motor deficits by self-report. Table I presents pertinent characteristics of the blind speakers. Twelve sighted adult subjects were also recorded and formed the control group. They all had perfect vision (20/20) or impaired vision cor- 
TABLE I. Characteristics of the 12 blind speakers.

\begin{tabular}{|c|c|c|c|c|c|}
\hline Subject & Gender & Age & Etiology of blindness & Vision at birth & Current vision \\
\hline DM & $\mathrm{F}$ & 48 & Retinitis pigmentosa & $\mathrm{U}^{\mathrm{a}}$ & $\begin{array}{l}\text { R.E. }^{b}=3 / 210 \\
\text { L.E. }^{\mathrm{c}}=0\end{array}$ \\
\hline FB & $\mathrm{F}$ & 40 & Congential cataract & $\mathrm{U}$ & $\begin{array}{c}\text { R.E. }=0 \\
\text { L.E. }=6 / 1260\end{array}$ \\
\hline SS & $\mathrm{F}$ & 26 & $\mathrm{U}$ & $\mathrm{U}$ & $\begin{array}{c}\mathrm{U} \\
\text { (total blindness) }\end{array}$ \\
\hline $\mathrm{CP}$ & M & 52 & Optic atrophy & Total blindness & $\begin{array}{l}\text { R.E. }=0 \\
\text { L.E. }=0\end{array}$ \\
\hline $\mathrm{SN}$ & M & 40 & Detachment of the retina & $\mathrm{U}$ & $\begin{array}{l}\text { R.E. }=2 / 180 \\
\text { L.E. }=2 / 105\end{array}$ \\
\hline YL & M & 42 & $\begin{array}{l}\text { Congential cataract et } \\
\text { Congential glaucoma }\end{array}$ & $\mathrm{U}$ & $\begin{array}{c}\mathrm{U} \\
\text { (total blindness) }\end{array}$ \\
\hline MAR & M & 36 & Retinitis pigmentosa & Total blindness & $\begin{array}{l}\text { R.E. }=20 / 400 \\
\text { L.E. }=20 / 400\end{array}$ \\
\hline $\mathrm{AB}$ & M & 52 & Congential cataract & Total blindness & $\begin{array}{l}\text { R.E. }=3 / 180 \\
\text { L.E. }=2 / 180\end{array}$ \\
\hline IM & $\mathrm{F}$ & 51 & Retinitis pigmentosa & Total blindness & $\begin{array}{l}\text { R.E. }=2 / 400 \\
\text { L.E. }=2 / 400\end{array}$ \\
\hline FM & $\mathrm{F}$ & 45 & Congential cataract & Total blindness & $\begin{array}{c}\mathrm{U} \\
\text { (total blindness) }\end{array}$ \\
\hline $\mathrm{JL}$ & $\mathrm{F}$ & 52 & Retinitis pigmentosa & $\mathrm{U}$ & $\begin{array}{c}\mathrm{U} \\
\text { (total blindness) }\end{array}$ \\
\hline MD & M & 42 & Retinitis pigmentosa & Total blindness & $\begin{array}{c}\mathrm{U} \\
\text { (total blindness) }\end{array}$ \\
\hline
\end{tabular}

${ }^{\mathrm{a}}$ Undetermind.

${ }^{\mathrm{b}}$ Right eye.

${ }^{\mathrm{c}}$ Left eye.

rected by lenses, resulting in near-perfect vision (according to self-report). The control subjects ranged in age from 22 to 39 years (mean: 33). Despite the mean age difference between the groups, it is unlikely to influence the results, as small age-related changes in perception and production that may exist tend to emerge at more advanced ages. Moreover, as will be seen, in this instance, the older (blind) group ultimately demonstrates more accurate auditory discrimination scores. All subjects passed a 20-dB HL pure-tone screening at 500, 1000, 2000, 4000, and $8000 \mathrm{~Hz}$.

\section{B. Experiment I: Perception}

Five sets of vowels (including the first five formants) ranging from /i/ to /e/, /e/ to / $/$ /, / / / to /a/, /y/ to /u/, and /i/ to /y/ (all phonemically contrastive) were synthesized using the variable linear articulatory model (Boë and Maeda, 1997), which is based on Maeda's model (Maeda, 1979). Whereas one might suggest that the use of synthetic stimuli does not adequately reflect natural speech processing, it represents the most appropriate means of controlling the precise acoustic differences across the stimuli. The five continua corresponded to the three phonological features along which French oral vowels are produced: height (/i/ vs /e/, /e/ vs / $\varepsilon$ /, and $/ \varepsilon / \mathrm{vs} / \mathrm{a} /)$, place of articulation $(/ \mathrm{y} / \mathrm{vs} / \mathrm{u} /)$, and rounding (/i/ vs /y/). Formant values of the end-point stimuli for each of the three continua, listed in Table II, were those used in previous perceptual studies with similar synthesized stimuli (Ménard et al., 2002; Ménard et al., 2004). Formant bandwidths for the five formants were calculated based on an analog simulation (Badin and Fant, 1984). Several versions of the five continua were created based on different steps between adjacent stimuli. Those stimuli were submitted to two native Canadian-French-speaking judges in order to determine the version that would maximally avoid ceiling effects while yielding scores above chance level (good discrimination functions). For the /i/ vs /e/ continuum, five stimuli were created between the end-points at equally stepped F1 (0.22 bark/20.1 mel), F2 (0.08 bark/7.4 mel), and F3 (0.34 bark/30.7 mel) distances. For the /e/ vs / $/ \varepsilon /$ continuum, five stimuli were also synthesized; F1 values be-

TABLE II. Formant $\left(F_{i}\right)$ and bandwidth $\left(B_{i}\right)$ values, in hertz, of end-point stimuli /i/, /e/, /y/, and /u/ synthesized for the perceptual experiment.

\begin{tabular}{|c|c|c|c|c|c|c|c|c|c|c|}
\hline Vowel & $\mathrm{F} 1$ & $\mathrm{~F} 2$ & $\mathrm{~F} 3$ & $\mathrm{~F} 4$ & F5 & B1 & $\mathrm{B} 2$ & B3 & B4 & B5 \\
\hline /i/ & 236 & 2062 & 3372 & 3466 & 5000 & 78 & 13 & 61 & 154 & 154 \\
\hline le/ & 372 & 1918 & 2501 & 3466 & 5000 & 78 & 13 & 61 & 154 & 154 \\
\hline $\mid \varepsilon /$ & 492 & 1676 & 2445 & 3610 & 5000 & 48 & 40 & 148 & 67 & 67 \\
\hline /a/ & 711 & 1234 & 2311 & 3695 & 5000 & 37 & 57 & 71 & 98 & 98 \\
\hline$/ y /$ & 236 & 1757 & 2062 & 3294 & 5000 & 88 & 40 & 19 & 19 & 19 \\
\hline$/ \mathrm{u} /$ & 236 & 705 & 2062 & 3294 & 5000 & 88 & 40 & 19 & 19 & 19 \\
\hline
\end{tabular}


tween stimuli differed by 0.18 bark $(16.5 \mathrm{mel})$, and F2 values varied by 0.15 bark ( $13.8 \mathrm{mel})$. Regarding the $/ \varepsilon /$ vs /a/ contrast, eight stimuli were created by varying $\mathrm{F} 1$ and $\mathrm{F} 2$ in equal steps (0.19 bark/17.4 mel and 0.22 bark/20.1 mel). As a result, seven stimuli (including end-points) were created for the /i/-/e/ dimension, seven for the /e/-/ / / continuum, and ten for the $/ \varepsilon /-/ / a /$ continuum. The $/ y /-/ u /$ continuum was represented by 22 stimuli, spaced in F2 by 0.26 bark (23.6 mel). The rounding continuum, corresponding to the /i/-/y/ dimension, was represented by seven stimuli, equally stepped in F2 (0.18 bark/16.5 mel) and F3 (0.52 bark/46.3 mel). A cascade formant synthesizer was excited by a glottal waveform generated by the Liljencrants-Fant source model. The resulting signal was digitized at $22 \mathrm{kHz}$ and was $600 \mathrm{~ms}$ long. A fall-rise amplitude contour was applied to the signal. The F0 values were $110 \mathrm{~Hz}$.

Stimuli from the five continua were presented to each of the subjects in a discrimination task. A classic AXB design was used, with an interstimulus interval of $500 \mathrm{~ms}$. Stimuli were grouped in triads where the first and the third were one step apart on the synthesized continuum, and the second was the same as either the first stimulus or the third one. After each triad was played, the subject had to decide whether the second stimulus was the same as the first or the third. Each triad was also presented in BXA form, where the order of the first and third stimuli was reversed. Each triad was repeated twice, in each order (AXB and BXA), yielding a total of four repetitions for a given pair of stimuli. All stimuli were randomized across listeners.

\section{Experiment II: Production}

\section{Procedure}

Each participant in the auditory discrimination test also served as a subject in a production task. Ten repetitions of the ten French oral vowels /i y u e $\varnothing$ o $\varepsilon \propto \rho$ a/ were elicited from each speaker, in random order, in the following context: "V comme WORD" ("V as in WORD"), where V is one of the ten vowels mentioned above, and WORD is a French word with this vowel in initial position. Only the initial isolated, long, and sustained $\mathrm{V}$ was analyzed (not the $\mathrm{V}$ produced in the word context). All speakers repeated the sequence after hearing an adult speaker utter it. For the sighted group, no visual input was provided. The speech signals were recorded in a sound booth with a high-quality tabletop microphone (Shure SM-84) at a 15- to 20-cm distance from the subject's lips and digitized at $44100 \mathrm{~Hz}$ by a digital audio tape recorder (DAT TASCAM DA-P1). Signals were then downsampled to $22050 \mathrm{~Hz}$ after low-pass filtering (cutoff frequency of $10000 \mathrm{~Hz}$ ). For each of the ten vowel repetitions, the first three formant frequencies were then extracted for each vowel, using the Linear Predictive Coding algorithm integrated in the PRAAT speech analysis program (Boersma and Weenink, 2007). The number of poles varied from 10 to 14 in the range of parameters used by Lee $e t$ al. (1999) and Hillenbrand et al. (1995). A 14-ms Hamming window was used with a pre-emphasis factor of 0.98 (preemphasis from $50 \mathrm{~Hz}$ for a sampling frequency of $22050 \mathrm{~Hz}$ ). Formant measurement errors were detected by comparing, for each vowel, the automatically extracted formant values overlaid on a wide-band spectrogram with a spectral slice obtained by an Fast Fourier Transform analysis with a Hamming window. When major discrepancies were observed either (i) between the overlaid formant values and the spectrogram or (ii) between the overlaid formant values and the spectral slice, the prediction order of the automatic detection algorithm was readjusted and the analysis was performed again. Fundamental frequency values (F0) were extracted using an autocorrelation algorithm. The formant frequencies were then converted to the mel scale because this scale models the ear's integration of frequency according to the following formula: $F_{\mathrm{mel}}=550 \ln \left(1+F_{\mathrm{Hz}} / 550\right)$.

\section{Data analysis}

At the perceptual level, for each triad, the number of correct responses, referred to as the discrimination score, was computed. The highest discrimination score obtained for the triads of a given continuum will be referred to as the peak discrimination score. In such a perceptual task, this score reflects auditory discrimination abilities at the category boundary between two stimuli. Repeated-measures Analysis of Variance (ANOVAs) were then carried out with peak discrimination scores as the dependent variable, vowel continuum (/i/ vs /e/, /e/ vs $/ \varepsilon /, / \varepsilon /$ vs /a/, /i/ vs /y/, or /y/vs /u/) as the within-subject factor, and subject group (blind or sighted) as the between-subject factor. Since the scores were relatively high, with data ranging from $62.5 \%$ to $100 \%$, ceiling effects were obtained, resulting in right-skewed distributions. Scores were thus transformed into logarithmic-based scales.

At the production level, the stimuli produced were represented in the traditional F1 vs F2 vs F3 space in mels. This three-dimensional space was used rather than the twodimensional F1 vs F2 space to account for possible shifts in formant-cavity affiliations across subjects, yielding greater contrast in the F2 vs F3 space between two vowel categories, than in the F1 vs F2 space. This is the case, for instance, for the /i/ vs /y/ rounding contrast in French (Schwartz et al., 1993).

Following Lane et al. (2001) and Ménard et al. (2007), among others, measures of contrast distances between vowel categories were computed. Those parameters have already been used in studies of speech produced by cochlear implant users as measures of produced contrasts (Lane et al., 2001; Ménard et al., 2007). In such studies, for a given vowel contrast, it is assumed that greater contrast distance between vowels reflects greater control and precision in the ability to produce this vowel contrast.

For each participant, Euclidean distances were first calculated between the mean F1, F2, and F3 values in mels (for each of the vowels) for all possible pairs of vowels in the acoustic space. Euclidean distances are more appropriate for cross-speaker comparisons than raw F1, F2, and F3 data since the latter are closely related to vocal-tract morphology and are speaker dependent. The first dependent variable at the production level consisted of the Euclidean distances for the vowels corresponding to the stimuli used in the perceptual task (/i/ vs /e/, /e/ vs / / /, / / / vs /a/, /i/ vs /y/, and /y/ vs 

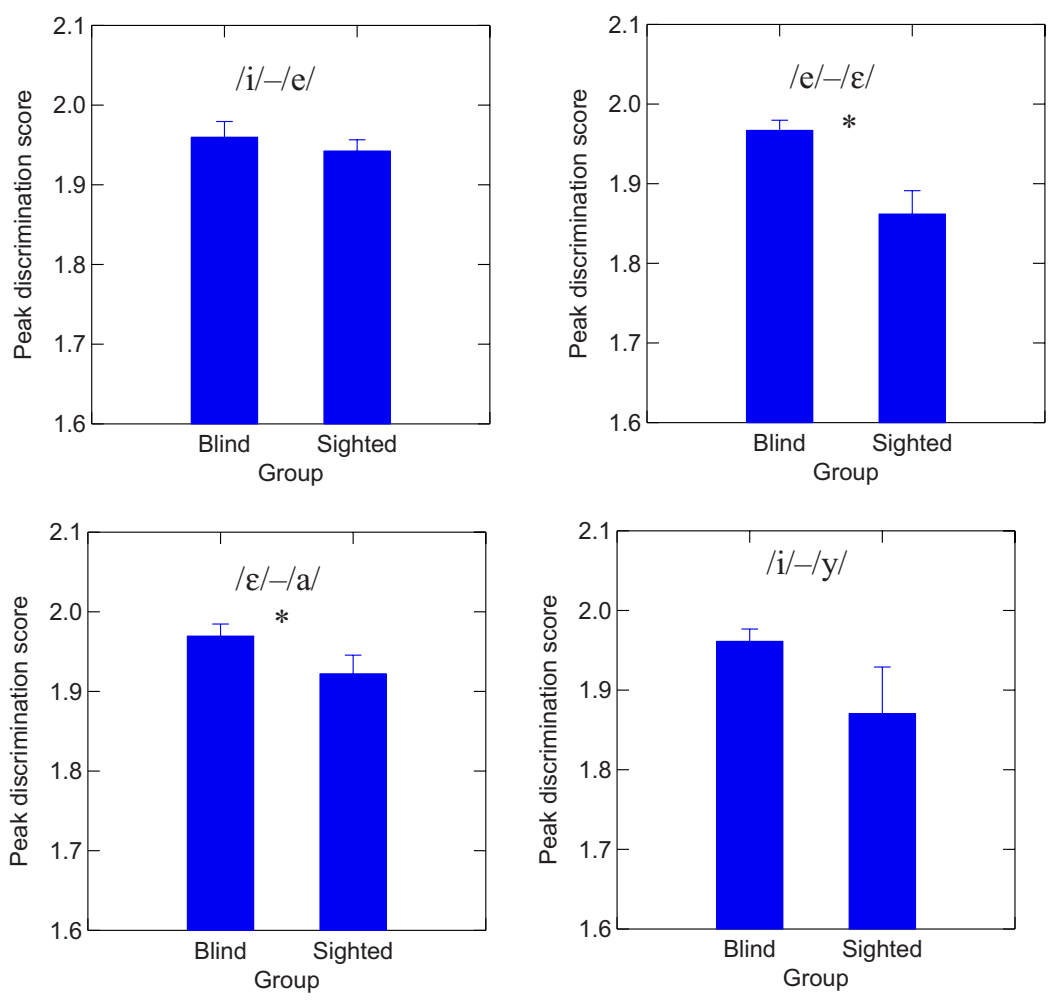

FIG. 1. (Color online) Average peak discrimination scores for both speaker groups for the five vowel contrasts: /i///e/ (upper left panel),/e/-/E/ (upper right panel), $/ \varepsilon /-/ \mathrm{a} /$ (middle left panel), /i///y/ (middle right panel), and /y/-/u/ (lower left panel). Error bars are one standard error of the mean.

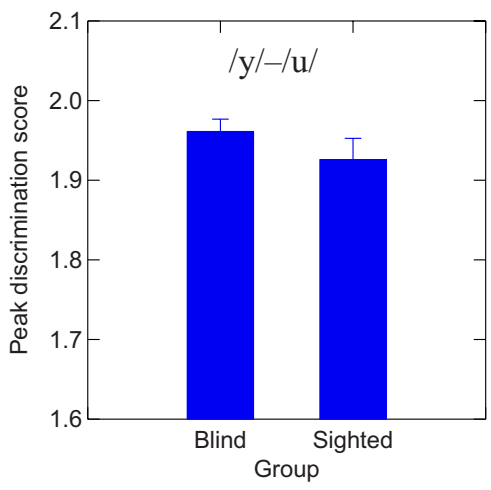

$/ \mathrm{u} /$ ). The second dependent variable was the average vowel spacing (AVS) (Lane et al., 2001) defined as the average of all Euclidean distances (including those between vowel pairs not used as stimuli at the perceptual level). Unlike produced Euclidean distances, AVS provides a global measure of produced vowel contrasts. Repeated-measures ANOVAs were then carried out on the data with subject group (blind or sighted) as the between-subject factor. Vowel continuum (/i/ vs /e/, /e/ vs / $\varepsilon /$, / $\varepsilon /$ vs /a/, /i/ vs /y/, and /y/ vs /u/) was the within-subject factor for the first dependent variable (Euclidean distances between vowels).

To further investigate the link between production and perception, multiple regression analyses were performed. For each of the five vowel continua, 12 data points (one for each speaker) were used. The dependent variable was produced Euclidean distance, in mels, and the independent variables were peak discrimination scores and speaker group.

\section{RESULTS}

\section{A. Perception}

Average peak discrimination scores for the three continua related to vowel height, rounding, and place of articu- lation for sighted and blind speakers are plotted in Fig. 1

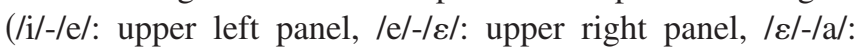
middle left panel, /i/-/y/: middle right panel, and $/ \mathrm{y} /-/ \mathrm{u} /$ : lower left panel). As Fig. 1 shows, all participants had good discrimination acuity, as revealed by the rather high average values for the peak discrimination scores. A repeatedmeasures ANOVA with peak discrimination scores as the dependent variable, speaker group (sighted or blind) as the between-subject variable, and vowel contrast (/i/-/e/, /e/-/ $/$ /, / $\varepsilon /-/ a /, / \mathrm{i} /-/ y /, / \mathrm{y} /-/ \mathrm{u} /$ ) as the within-subject variable did not reveal any significant main effects of speaker group or vowel contrast. However, a significant interaction of speaker group and vowel contrast was found $[F(4,88)=2.51 ; p<0.05]$. Post hoc tests (Tukey) showed that blind speakers had significantly higher peak discrimination scores than sighted speakers for the $/ \mathrm{e} /-/ \varepsilon /$ contrast $[F(1,22)=15.60 ; p<0.05]$ as well as for the $/ \varepsilon /-/ \mathrm{a} /$ contrast $[F(1,22)=5.12 ; p<0.05]$. The difference in peak discrimination scores for the /i/-/y/ continuum did not reach significance $(p<0.07)$ but the observed pattern is similar to the significant one noted for the $/ \mathrm{e} /-/ \varepsilon /$ and $/ \varepsilon /-/ a /$ contrasts, with blind speakers having higher scores than sighted speakers. A closer examination of the 

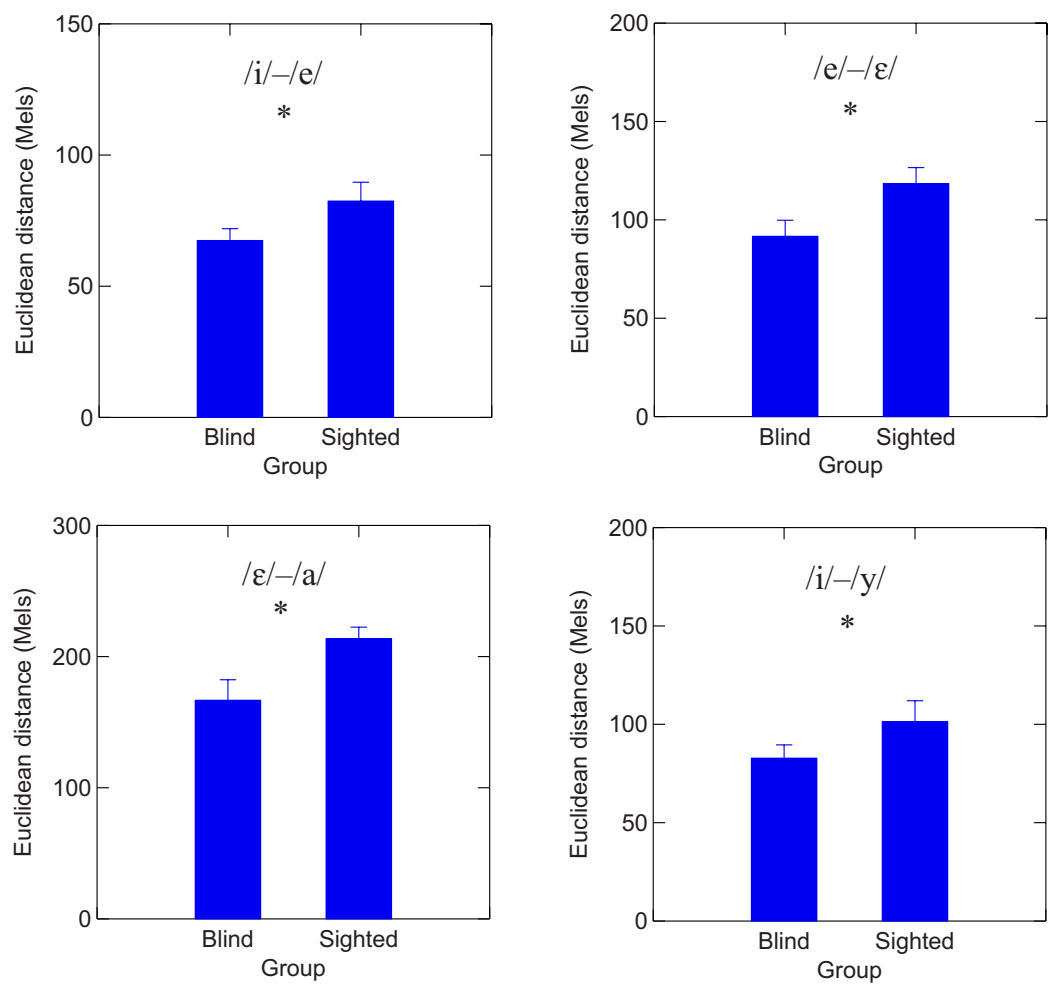

FIG. 2. (Color online) Average produced Euclidean distances for both speaker groups for the five vowel contrasts: /i/-/e/ (upper left panel),/e/-/E/ (upper right panel), / $\varepsilon /-/ \mathrm{a} /$ (middle left panel), /i/-/y/ (middle right panel), and /y/-/u/ (lower left panel). Error bars are one standard error of the mean.

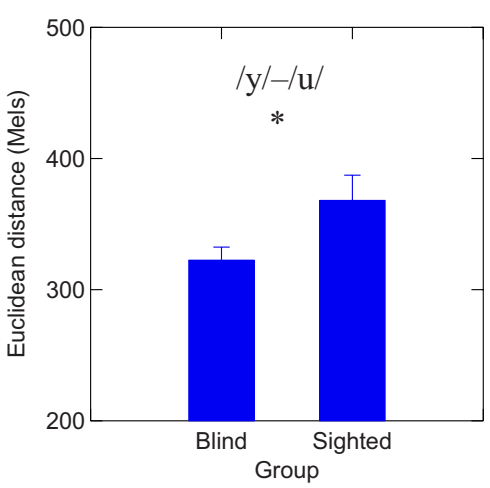

data revealed that standard deviation values (as suggested by the size of the error bars in Fig. 1) are higher for sighted subjects than for blind subjects. This pattern is mainly due to the perfect discrimination score $(100 \%)$ of two sighted subjects, the remaining ten subjects having peak discrimination scores close to $80 \%$. A ceiling effect is probably involved here, preventing the tendency toward higher discrimination scores for blind than sighted subjects from reaching significance.

\section{B. Production}

The average Euclidean distances measured for the /i/-/e/, /e/-/ $\varepsilon /, / \varepsilon /-/ \mathrm{a} /$, /i/-/y/, and /y/-/u/ produced contrasts are plotted in Fig. 2. Error bars represent one standard error of the mean. A repeated-measures ANOVA with Euclidean distance as the dependent variable, speaker group (sighted or blind) as the between-subject variable, and vowel contrast (/i/-/e/, /e/-/ $\varepsilon /, / \varepsilon /-/ \mathrm{a} /, / \mathrm{i} /-/ \mathrm{y} /, / \mathrm{y} /-/ \mathrm{u} /$ ) as the within-subject variable revealed a significant main effect of speaker group, with blind subjects having smaller contrast distances than sighted subjects $[F(1,22)=14.33 ; p<0.05]$. A significant effect of vowel contrast was also observed, with the distances between $/ \mathrm{y} /$ and $/ \mathrm{u} /$ being greater than the distances between the other vowel pairs $[F(4,88)=12.43 ; p<0.05]$. This result reflects the organization of the French vowel space, the rounded back vowel /u/ having no unrounded counterpart (in contrast to the front rounded /y/ vs the front unrounded /i/). The analysis did not reveal any significant effect of the interaction between the group variable and the vowel contrast variable.

Figure 3 plots the average AVS values for sighted speakers and blind speakers. A one-way ANOVA performed on this data set with speaker group (sighted or blind) as the between-subject factor revealed a significant effect of speaker group $[F(1,20)=6.20 ; p<0.05]$. Sighted speakers produced larger contrast distances between vowel categories (AVS) than blind speakers.

\section{Relations between production and perception}

For each of the five vowel continua, in Fig. 4, data are plotted in "production-perception" space (/i/-/e/: upper left

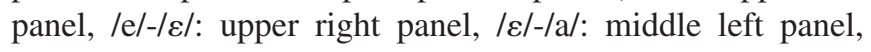




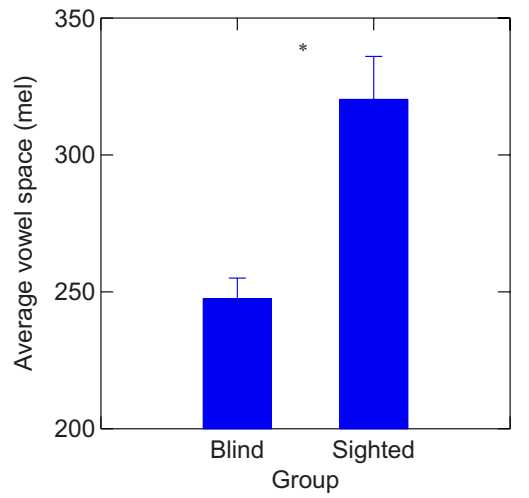

FIG. 3. (Color online) AVS in the F1 vs F2 vs F3 space, in mels, for both speaker groups.

/i/-/y/: middle right panel, and /y/-/u/: lower left panel). The production axis (x-axis) corresponds to the produced contrast distance (Euclidean distance), in mels, for each speaker. The perception dimension (y-axis) is the corresponding value of
TABLE III. Values of beta weights $(B)$ from multiple regression analyses for each vowel contrast. Dependent variables: produced Euclidean distances; independent variables: subject group and peak discrimination scores (* $=$ significant at $p<0.05$ ).

\begin{tabular}{ccc}
\hline \hline Contrast & $B$ group & $B$ peak discrimination score \\
\hline$/ \mathrm{i} /-/ \mathrm{e} /$ & 0.35 & -0.06 \\
$/ \mathrm{e} /-/ \varepsilon /$ & $0.73^{*}$ & $0.46^{*}$ \\
$/ \varepsilon /-/ \mathrm{a} /$ & $0.57^{*}$ & 0.27 \\
$/ \mathrm{i} / / \mathrm{y} /$ & 0.21 & -0.27 \\
$/ \mathrm{y} /-\mathrm{u} /$ & $0.44^{*}$ & 0.07 \\
\hline \hline
\end{tabular}

the peak discrimination score for each speaker. As a result, 12 data points are represented within each subject group (blind and sighted) and for each vowel continuum. Results of multiple regression analyses are presented in Table III. Beta weights, given for each of the independent variables, are interpretable in terms of magnitude of influence of a variable on the produced contrast distance. As shown in Table III, the
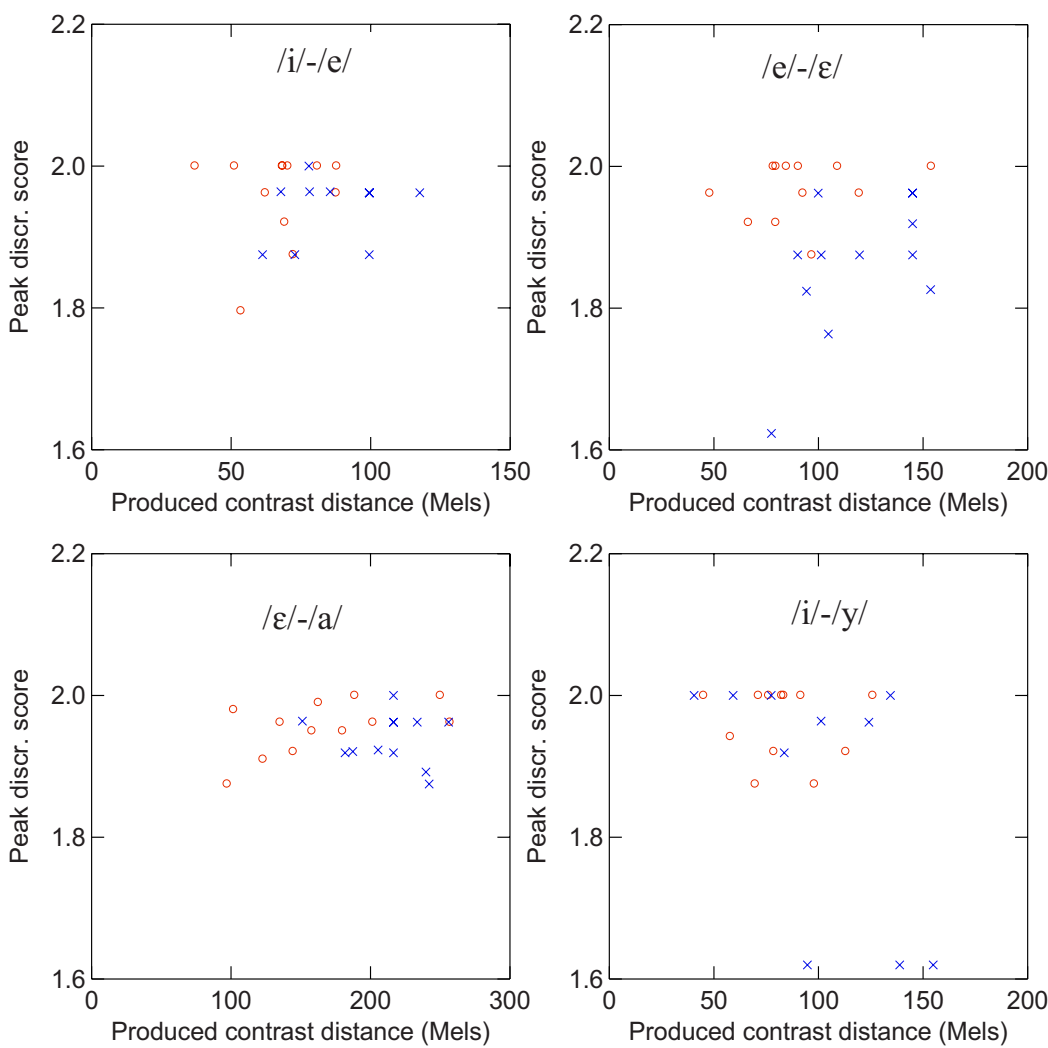

FIG. 4. (Color online) Average produced Euclidean distances in relation with peak discrimination scores for both speaker groups for the five vowel contrasts: /i/-/e/ (upper left panel), /e/-/ / / (upper right panel), / $\varepsilon /-/ \mathrm{a} /$ (middle left panel), /i/-/y/ (middle right panel), and /y/-/u/ (lower left panel). Results of multiple regression analyses are shown in Table III.

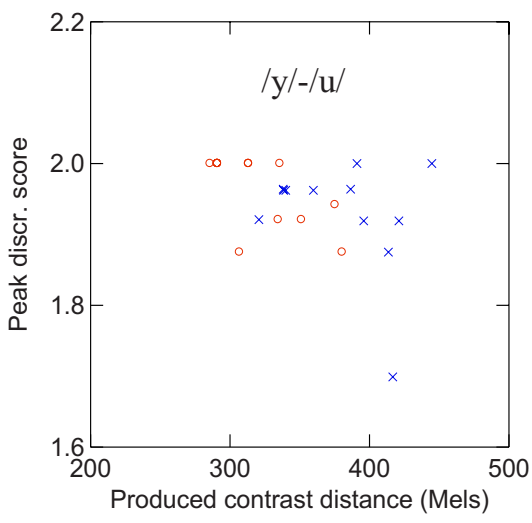

Speaker group

$\circ$ Blind

$\times$ Sighted 
effect of the group variable is significant for $/ \mathrm{e} /-/ \varepsilon /, / \varepsilon /-/ \mathrm{a} /$, and $/ y /-/ u /$. The effect of peak discrimination score is significant only for the $/ \mathrm{e} /-/ \varepsilon /$ contrast, and its beta weight is lower than the one calculated for the group variable. These results suggest that lack of visual input has a stronger influence on the production of the vowel contrasts than does perceptual acuity for those contrasts, although the two co-vary to some degree.

\section{DISCUSSION}

In order to assess the role of visual deprivation on auditory perception and production of French vowels, discrimination tasks and acoustic recordings were conducted with congenitally blind subjects and sighted control subjects. Significant effects of speaker groups were found in both tasks (although for only a subset of vowel discrimination contrasts), confirming the importance of the role of visual experience in speech perception and production.

\section{A. Auditory perception and produced contrast distances}

At the perceptual level, the results of these experiments showed that congenitally blind adult speakers have more accurate auditory discrimination abilities than sighted adult speakers for some French oral vowels. Indeed, in AXB discrimination tasks performed on synthesized continua, blind speakers had significantly higher peak discrimination scores than sighted speakers for two continua $(/ \mathrm{e} /-/ \varepsilon /$ and $/ \varepsilon /-/ \mathrm{a} /)$, and the same tendency almost reached significance for a third continuum $(/ \mathrm{i} /-/ \mathrm{y} /)$. The fact that discrimination scores were higher only for two out of five continua is likely due to a ceiling effect. This result confirms those of earlier studies showing that blind speakers have better auditory acuity than sighted speakers (Lucas, 1984; Hugdahl et al., 2004; Gougoux et al., 2004; Doucet et al., 2005). Those contrasts are related to both height and rounding features, two dimensions that are highly associated with visual correlates at the perceptual level in French. Perhaps blind listeners are more attuned to the acoustic properties of these contrasts because they cannot rely on the additional visual cues. Although it is possible that the blind listeners had more experience with synthetic speech in their lifetimes, given the high accuracy levels of both groups and the fact that our findings are consistent with previous investigations, it is unlikely that such experience (if present) contributed significantly to the results.

At the production level, contrast distances, measured by the value of AVS, were significantly higher for sighted speakers than for blind speakers. According to Perkell et al. (2004), speakers who are better able to discriminate auditorily between phoneme categories will tend to produce larger contrast distances between categories. Thus, blind speakers, who have more accurate auditory discrimination abilities, would be expected to produce larger AVS values. Interestingly, the opposite pattern was observed, suggesting that the effects of absence of visual feedback on vowel contrasts may be larger than the effects of auditory acuity. [Of course, additional unrelated factors, such as differences in language acquisition and socialization, educational environment, etc., may also play a role (e.g., Andersen et al., 1993).] This result is somewhat similar to that reported by Perkell and colleagues (Perkell et al., 2004; Ménard et al., 2007) on postlingually deaf speakers of American English with cochlear implants. Although several differences exist between the two sets of studies, in the Perkell group's studies, absence of auditory feedback yielded reduced contrast distances between categories in acoustic space, as revealed by reduced AVS values. In the present study, absence of visual feedback from birth was found to lead to similar results.

The present results indicate that the absence of visual input may contribute to more accurate auditory discrimination scores, suggesting that the internal phonemic sensory goals may be more distinct (see, e.g., Guenther et al., 2006). However, the contrast distances (AVS) measured in the production task were smaller for speakers with visual impairments compared to sighted speakers, suggesting that visual cues play an important part in shaping speech goals.

The findings are consistent with recent behavioral and neuroimaging investigations which have supported a close link between perception and production (e.g., Fadiga et al., 2002; Watkins et al., 2003; Wilson et al., 2004; Sams et al., 2005; Pulvermuller et al., 2006; Gentilucci and Bernardis, 2007; Meister et al., 2007; Skipper et al., 2007; Tourville et $a l ., 2008)$. That is, despite the absence of a strong statistical relationship between the auditory-perceptual and production data, we interpret the results to suggest an important link between the perceptual representation (developed on the basis of both auditory and visual cues) and production patterns. Further studies conducted with a greater number of subjects will seek to investigate whether auditory acuity in blind speakers is specifically related to the amount of acoustic contrasts produced by those speakers.

\section{ACKNOWLEDGMENTS}

This work was supported by the Social Sciences and Humanities Research Council of Canada and the Natural Sciences and Engineering Research Council of Canada. We are grateful to Pascal Perrier for useful comments on earlier versions of this paper. Thanks to Zofia Laubitz for copy-editing the paper.

Andersen, E., Dunlea, A., and Kekelis, L. (1993). "The impact of input: Language acquisition in the visually impaired," First Lang. 13, 23-50.

Andersson, U., Lyxell, B., Rönnberg, J., and Spens, K. E. (2001). "Cognitive correlates of visual speech understanding in hearing-impaired individuals," Journal of Deaf Studies and Deaf Education 6, 103-116.

Badin, P., and Fant, G. (1984). "Notes on vocal tract computation," Speech Transm. Lab. Q. Prog. Status Rep. 2-3, 53-108.

Bell-Berti, F., Raphael, L. J., Pisoni, D. B., and Sawusch, J. R. (1979). "Some relationships between speech production and perception," Phonetica 36, 373-383.

Bernstein, L. E., Auer, E. T., Jr., and Tucker, P. E. (2001). "Enhanced speechreading in deaf adults: Can short-term training/practice close the gap for hearing adults $₫$," J. Speech Lang. Hear. Res. 44, 5-18.

Bernstein, L. E., Demorest, M. E., and Tucker, P. E. (2000). "Speech perception without hearing," Percept. Psychophys. 62, 233-252.

Boë, L.-J., and Maeda, S. (1997). "Modélisation de la croissance du conduit vocal. Espace vocalique des nouveaux-nés et des adultes. Conséquences pour l'ontogenèse et la phylogenèse," Journées d'Études Linguistiques: "La Voyelle dans Tous ces États," 1, 98-105. 
Boersma, P., and Weenink, D. (2007). PRAAT, Version 4.4.07, www.praat.org (Last viewed February, 2007).

Bradlow, A. R., Pisoni, D. B., Akahane-Yamada, R., and Tohkura, Y. (1997). "Training Japanese listeners to identify English /r/ and /l/: IV. Some effects of perceptual learning on speech production," J. Acoust. Soc. Am. 101, 2299-2310.

Doucet, M.-E., Guillemot, J.-P., Lassonde, M., Gagné, J.-P., Leclerc, C., and Lepore, F. (2005). "Blind subjects process auditory spectral cues more efficiently than sighted individuals," Exp. Brain Res. 160, 194-202.

Elstner, W. (1983). "Abnormalities in the verbal communication of visuallyimpaired children," in Language Acquisition in the Blind Child, edited by A. E. Mills (Croom Helm, London), pp. 18-41.

Fadiga, L., Craighero, L., Buccino, G., and Rizzolatti, G. (2002). "Speech listening specifically modulates the excitability of tongue muscles: A TMS study," Eur. J. Neurosci. 15, 399-402.

Gentilucci, M., and Bernardis, P. (2007). "Imitation during phoneme production," Neuropsychologia 45, 608-615.

Göllesz, V. (1972). "Über die lippenartikulation der von geburt an blinden" ("About the lip articulation of the blind from birth"), in Papers in Interdisciplinary Speech Research. Speech Symposium, edited by S. Hirschberg, G. Y. Szépe, and E. Vass-Kovoics (Akadémiai Kiado, Budapest), pp. 85-91.

Gougoux, F., Lepore, F., Lassonde, M., Voss, P., Zatorre, R. J., and Belin, P. (2004). "Pitch discrimination in the early blind," Nature (London) 430, 309.

Grant, K. W., Walden, B. E., and Seitz, P. F. (1998). “Auditory-visual speech recognition by hearing-impaired subjects: Consonant recognition, sentence recognition, and auditory-visual integration," J. Acoust. Soc. Am. 103, 2677-2690.

Guenther, F. H., Ghosh, S. S., and Tourville, J. A. (2006). "Neural modeling and imaging of the cortical interactions underlying syllable production," Brain Lang 96, 280-301.

Hillenbrand, J., Getty, L. A., Clark, M. J., and Wheeler, K. (1995). “Acoustic characteristics of American English vowels," J. Acoust. Soc. Am. 97, 3099-3111.

Hugdahl, K., Ek, M., Rintee, T., Tuomainen, J., Haaral, C., and Hämäläinen, K. (2004). "Blind individuals show enhanced perceptual and attentional sensitivity for identification of speech sounds," Brain Res. Cognit. Brain Res. 19, 28-32.

Kuhl, P. K., and Meltzoff, A. N. (1982). "The bimodal perception of speech in infancy," Science 218, 1138-1141.

Lane, H., Matthies, M., Perkell, J., Vick, J., and Zandipour, M. (2001). "The effects of changes in hearing status in cochlear implant users on the acoustic vowel space and CV coarticulation,” J. Speech Lang. Hear. Res. 44, $552-563$.

Lee, S., Potamianos, A., and Narayanan, S. (1999). "Acoustics of children's speech. Developmental changes of temporal and spectral parameters," J. Acoust. Soc. Am. 105, 1455-1468.

Legerstee, M. (1990). "Infants use multimodal information to imitate speech sounds," Infant Behav. Dev. 13, 343-354.

Lewis, M. M. (1975). Infant Speech: A Study of the Beginnings of Language (Arno, New York).

Lucas, S. A. (1984). "Auditory discrimination and speech production in the blind child," Int. J. Rehabil. Res. 7, 74-76.

Maeda, S. (1979). "An articulatory model of the tongue based on a statistical analysis," J. Acoust. Soc. Am. 65, S22.

McGurk, H., and MacDonald, J. (1976). "Hearing lips and seeing voices," Nature (London) 264, 746-748.

Meister, I. G., Wilson, S. M., Deblieck, C., Wu, A. D., and Iacoboni, M. (2007). "The essential role of premotor cortex in speech perception," Curr. Biol. 17, 1692-1696.

Ménard, L., Polak, M., Denny, M., Lane, H., Matthies, M. L., Perkell, J. S., Burton, E., Marrone, N., Tiede, M., and Vick, J. (2007). "Interactions of speaking condition and auditory feedback on vowel production in postlingually deaf adults with cochlear implants," J. Acoust. Soc. Am. 121,
3790-3801

Ménard, L., Schwartz, J.-L., and Boë, L.-J. (2004). "Role of vocal tract morphology in speech development: Perceptual targets and sensorimotor maps for synthesized french vowels from birth to adulthood," J. Speech Lang. Hear. Res. 47, 1059-1080.

Ménard, L., Schwartz, J.-L., Boë, L.-J., Kandel, S., and Vallée, N. (2002). "Auditory normalization of French vowels synthesized by an articulatory model simulating growth from birth to adulthood," J. Acoust. Soc. Am. 111, 1892-1905.

Miller, J. (1992). "Diderot reconsidered: Visual impairment and auditory compensation,” J. Vis. Impair. Blind. 86, 206-210.

Mills, A. E. (1987). "The development of phonology in the blind child," in Hearing by Eye: The Psychology of Lip-Reading, edited by B. Dodd and R. Campbell (Lawrence Erlbaum Associates, London), pp. 145-163.

Newman, R. S. (2003). "Using links between speech perception and speech production to evaluate different acoustic metrics: A preliminary report," J. Acoust. Soc. Am. 113, 2850-2860.

Niemeyer, W., and Starlinger, I. (1981). "Do the blind hear better? Investigations on auditory processing in congenital or early acquired blindness. II. Central functions," Audiology 20, 510-515.

Perkell, J. S., Guenther, F. H., Lane, H., Matthies, M. L., Stockmann, E., Tiede, M., and Zandipour, M. (2004). “The distinctness of speakers' productions of vowel contrasts is related to their discrimination of the contrasts," J. Acoust. Soc. Am. 116, 2338-2344.

Pulvermuller, F., Huss, M., Kherif, F., Moscoso del Prado Martin, F., Hauk, O., and Shtyrov, Y. (2006). "Motor cortex maps articulatory features of speech sounds," Proc. Natl. Acad. Sci. U.S.A. 103, 7865-7870.

Reisberg, D., McLean, J., and Goldfield, A. (1987). "Easy to hear but hard to understand: A lip-reading advantage with intact auditory stimuli," in Hearing by Eye: The Psychology of Lip-Reading, edited by B. Dodd and R. Campbell (Lawrence Erlbaum Associates, Hillsdale, NJ), pp. 97-113.

Robert-Ribes, J., Schwartz, J. L., Lallouache, T., and Escudier, P. (1998). "Complementarity and synergy in bimodal speech: Auditory, visual and audiovisual identification of French oral vowels in noise," J. Acoust. Soc. Am. 103, 3677-3689.

Rvachew, S. (1994). "Speech perception training can facilitate sound production learning," J. Speech Hear. Res. 37, 347-357.

Sams, M., Mottonen, R., and Sihvonen, T. (2005). "Seeing and hearing others and oneself talk," Brain Res. Cognit. Brain Res. 23, 429-435.

Schwartz, J.-L., Beautemps, D., Abry, C., and Escudier, P. (1993). "Interindividual and cross-linguistic strategies for the production of the [i] vs. [y] contrast," J. Phonetics 21, 411-425.

Skipper, J. I., Van Wassenhove, V., Nusbaum, H. C., and Small, S. L. (2007). "Hearing lips and seeing voices: How cortical areas supporting speech production mediate audiovisual speech perception," Cereb. Cortex 17, 2387-2399.

Stankov, L., and Spilsbury, G. (1978). "The measurement of auditory abilities of blind, partially sighted, and sighted children," Appl. Psychol. Measure. 2, 491-503.

Starlinger, I., and Niemeyer, W. (1981). "Do the blind hear better? Investigations on auditory processing in congenital or early acquired blindness. I. Peripheral functions," Audiology 20, 503-509.

Tourville, J. A., Reilly, K. J., and Guenther, F. H. (2008). "Neural mechanisms underlying auditory feedback control of speech," Neuroimage $\mathbf{3 9}$, 1429-1443.

Vick, J., Lane, H., Perkell, J. S., Matthies, M. L., Gould, J., and Zandipour, M. (2001). "Covariation of cochlear implant users' perception and production of vowel contrasts and their identification by listeners with normal hearing," J. Speech Lang. Hear. Res. 44, 1257-1267.

Watkins, K. E., Strafella, A. P., and Paus, T. (2003). "Seeing and hearing speech excites the motor system involved in speech production," Neuropsychologia 41, 989-994.

Wilson, S. M., Saygin, A. P., Sereno, M. I., and Iacoboni, M. (2004). "Listening to speech activates motor areas involved in speech production," Nat. Neurosci. 7, 701-702. 